\title{
Histone deacetylase inhibitor enhances the anti-tumor effect of gemcitabine: A special reference to gene- expression microarray analysis
}

\author{
SHUICHI IWAHASHI, MITSUO SHIMADA, TOHRU UTSUNOMIYA, YUJI MORINE, SATORU IMURA, \\ TETSUYA IKEMOTO, HIROKI MORI, JUN HANAOKA and YU SAITO
}

Department of Surgery, Institute of Health Biosciences, The University of

Tokushima Graduate School, Tokushima 770-8503, Japan

Received April 27, 2011; Accepted June 1, 2011

DOI: 10.3892/or.2011.1407

\begin{abstract}
Histone deacetylase (HDAC) is strongly associated with epigenetic regulation and carcinogenesis, and its inhibitors induce the differentiation or apoptosis of cancer cells. Valproic acid (VPA) is one of the clinically available HDAC inhibitors. We investigated the anticancer effects of VPA in combination with gemcitabine (GEM) in the human cholangiocarcinoma cell line HuCCT1, and explored the mechanisms of the anticancer effects using microarray analysis. The anticancer effects of VPA or gemcitabine (GEM), and the effects of VPA combined with GEM, were studied by a cell proliferation assay. A microarray analysis was performed and the genes were picked up using GeneSpring GX11.5, followed by Ingenuity Pathways Analysis (IPA) and determination of gene expression by RT-PCR. GEM $(5 \mathrm{nM})$ and VPA $(0.5 \mathrm{mM})$ reduced proliferation by $23 \%$, which significantly augmented the anticancer effect of GEM alone or VPA alone $(\mathrm{P}<0.01)$. Using microarray analysis, 43 genes were identified with the comparison between the GEM group and the GEM plus VPA combination group. Interactions were identified between genes of the 'Cellular Development' network relevant to the differentiation of cancer cells using IPA. Furthermore, GEM combined with VPA up-regulated the HLA-DRA expression compared to the single agents $(\mathrm{P}<0.01)$. VPA augmented the effects of GEM by enhancing the gene network mainly including HLA-DRA, possibly through the modification of cancer cell differentiation.
\end{abstract}

\section{Introduction}

Cholangiocarcinoma is a cancer arising from bile duct epithelium. This cancer is one of the most difficult diseases to treat and

Correspondence to: Professor Mitsuo Shimada, Department of Surgery, Institute of Health Biosciences, The University of Tokushima, 3-18-15 Kuramoto-cho, Tokushima 770-8503, Japan E-mail:mshimada@clin.med.tokushima-u.ac.jp

Key words: cholangiocarcinoma, histone deacetylase inhibitor, valproic acid, microarray analysis, ingenuity pathways analysis no standard chemotherapy has been established $(1,2)$. Widely used chemotherapeutic regimens include gemcitabine (GEM), a nucleoside analogue of cytidine (2',2'-difluorodeoxycytidine; $\mathrm{dFdC}$ ), which has a response rate of $<30 \%$ (3). New agents and innovative therapeutic approaches are being investigated. Our group has researched the resistance of chemotherapy in biliary tract cancers (4).

Alterations in the epigenetic modulation of geneexpressions have been implicated in cancer development and progression. Histone acetylation is a post-translational modulation of the nucleosomal histones that affects chromatin structure and modulates gene-expression. Histone deacetylases (HDACs) comprise an ancient family of enzymes that play crucial roles in numerous biological processes (5) and expressions of HDACs are up-regulated in many tumor types $(6,7)$. We reported that the survival rate for pancreatic cancer patients that were HDAC1-positive was significantly lower than for those that were HDAC1-negative and HDAC1 was considered to be a promising therapeutic target in pancreatic cancer (8). HDAC inhibitors induce the differentiation or apoptosis of cancer cells $(9,10)$. Therefore, HDAC inhibitors are promising novel anticancer agents. In this study, we used valproic acid (VPA), which has been shown to have anticancer effects in various cancer models (11-13). However, no reports have focused on the alteration of gene-expression related to the anticancer effect of VPA in a bile duct cell line using microarray analysis.

The goal of this study was to investigate the anticancer effect of VPA in combination with GEM in a cholangiocarcinoma cell line and to identify the molecular mechanisms of the anticancer effect using microarray analysis.

\section{Materials and methods}

Cell lines and culture conditions. HuCCT1 (STR: http:// www2.brc.riken.jp/lab/cell/str_start.shtml?cell_no=RCB1960) was provided by the RIKEN BRC through the National Bio-Resource Project of the MEXT, Japan. All cell lines were grown in RPMI-1640, supplemented with $10 \%$ fetal bovine serum (FBS), $70 \mu \mathrm{g} / \mathrm{ml}$ penicillin, $100 \mu \mathrm{g} / \mathrm{ml}$ streptomycin (complete medium) and maintained at $37^{\circ} \mathrm{C}$ in a humidified 
incubator with $5 \% \mathrm{CO}_{2}$ in the air. The cells were maintained for no longer than 12 weeks after recovery from frozen stock.

Reagents. Valproic acid was purchased from Wako Pure Chemical Industries, Ltd. (Osaka, Japan), kept at $4^{\circ} \mathrm{C}$ and diluted in PBS as necessary at the time of use. Gemzar (gemcitabine hydrochloride) was purchased from Eli Lilly and Co. (Indianapolis, IN) and made fresh in $0.9 \% \mathrm{NaCl}$ on the day of use.

Cell proliferation assay. All of the tumor cells $\left(5 \times 10^{3}\right)$ were seeded into $38-\mathrm{mm}^{2}$ wells of flat-bottomed 96 -well plates in quadruplicate and allowed to adhere overnight. The spent medium was then removed and the cultures were re-fed with new medium (negative control) or medium, containing different concentrations of VPA and GEM. Incubation was continued for $72 \mathrm{~h}$ prior to adding the Cell Counting Kit-8. After $2 \mathrm{~h}$, the optical density was measured at $450 \mathrm{~nm}$ with a microplate reader (Multiskan JX; Lab Systems).

Microarray analysis. Total RNA was isolated from both the stimulated and unstimulated cells (HuCCT1) using the RNeasy Mini kit (Qiagen, Valencia, CA). Relative purity was examined using an Agilent 2100 Bioanalyzer (Agilent Technologies, Santa Clara, CA, USA). RNA expression was analyzed using the GeneChip ${ }^{\circledR}$ Human Gene 1.0 ST Array (Affymetrix, Santa Clara, CA, USA), containing 28,869 oligonucleotide probes for known and unknown genes. First strand cDNA was synthesized from $300 \mathrm{ng}$ of total RNA by using the GeneChip Whole Transcript (WT) cDNA Synthesis and Amplification kit (Affymetrix) according to the manufacturer's instructions. cRNA $(10 \mu \mathrm{g})$ was input into the second-cycle cDNA reaction. cDNA was fragmented and end-labeled with the GeneChip WT Terminal Labeling kit (Affymetrix). Approximately $5.5 \mu \mathrm{g}$ of fragmented and labeled DNA target was hybridized to the Affymetrix GeneChip Human Gene 1.0 ST Array at $45^{\circ} \mathrm{C}$ for $17 \mathrm{~h}$ in a GeneChip Hybridization Oven 640 (Affymetrix) according to the manufacturer's recommendation. Hybridized arrays were washed and stained on a GeneChip Fluidics Station 450 and scanned on a GeneChip Scanner 3000 7G (Affymetrix) and CEL files were then generated for each array. The microarray data were normalized by the GeneSpring GX 11.5 software (Agilent). The cut-off value was set at 0.5-2.0 for the ratio (>2.0, up-regulation; 0.5-2.0, no change; $<0.5$, down-regulation). The complete datasets were deposited in the Gene Expression Omnibus database (accession no. GSE27036).

Gene Ontology (GO) was analyzed using the GeneSpring GX 11.5 software (Agilent) and a P-value $<0.05$ was used for significance. The Ingenuity Pathway Analysis (IPA) 8.7 (http://www.ingenuity.com) was then used to determine the functional pathways associated with the set of differentially expressed genes between genotypes. IPA utilizes the knowledge in the literature about biological interactions among genes and proteins.

$R N A$ isolation and quantitative real-time RT-PCR. The extracted RNA was reverse transcribed with the High Capacity cDNA Reverse Transcription Kit (Applied Biosystems). Quantitative real-time RT-PCR was performed using the Applied Biosystems 7500 Real-Time PCR System, TaqMan Gene Expression
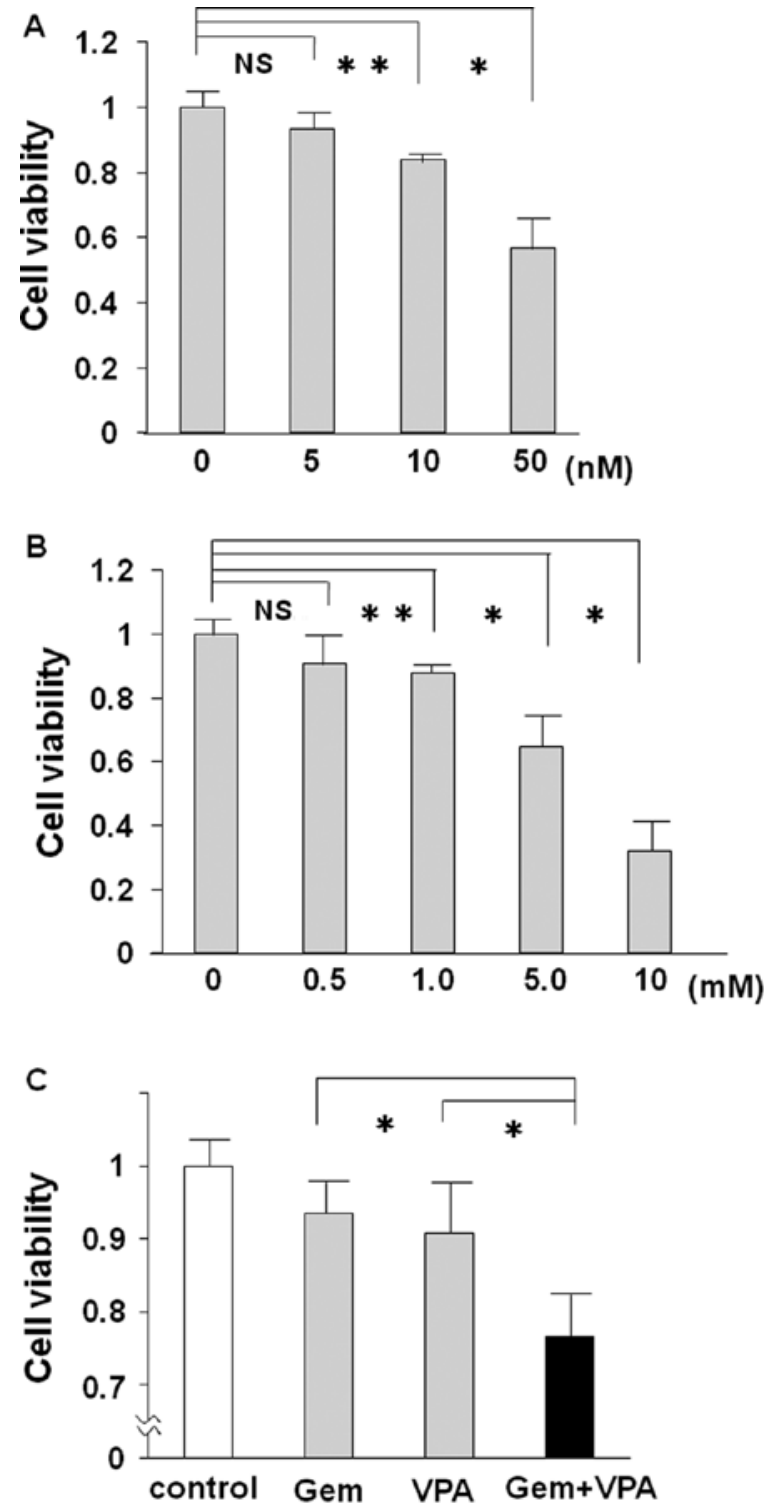

Figure 1. The effect of GEM (A), VPA (B) and the combination of GEM $(5 \mathrm{nM})$ and VPA $(0.5 \mathrm{mM})(\mathrm{C})$ on inhibiting cell proliferation of the human cholangiocarcinoma cell line, HuCCT1 ${ }^{* *} \mathrm{P}<0.05$; ${ }^{*} \mathrm{P}<0.01$; NS, not significant.

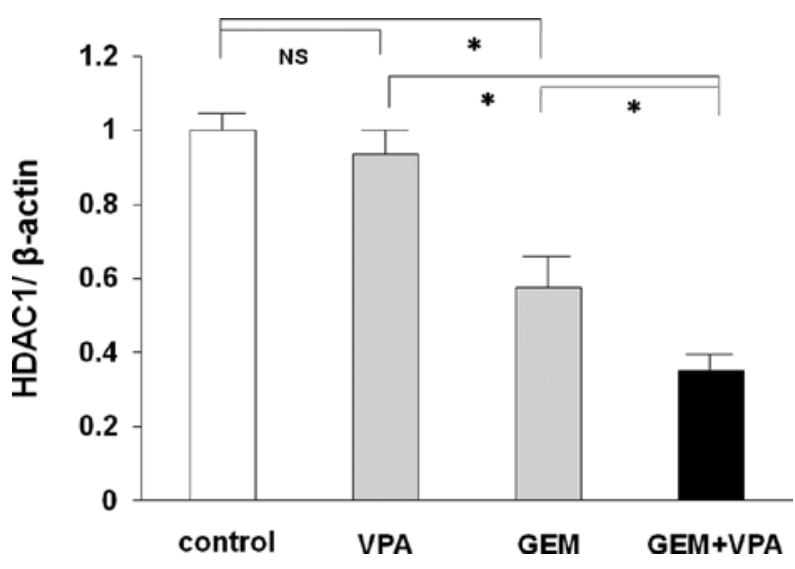

Figure 2. The gene-expressions of HDAC1 of human cholangiocarcinoma cell line, HuCCT1, in the combination of GEM $(5 \mathrm{nM})$ and VPA $(0.5 \mathrm{mM})$ ${ }^{*} \mathrm{P}<0.01$. 


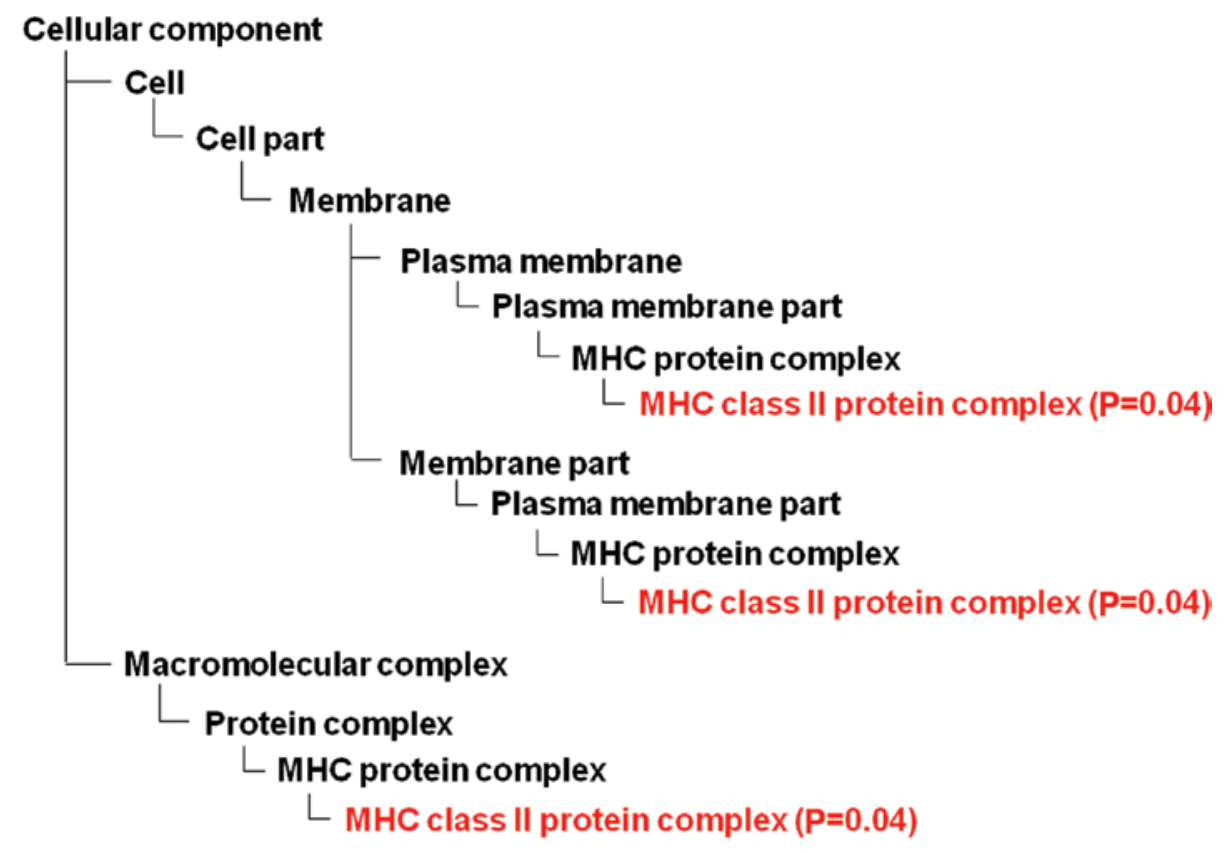

Figure 3. GO analysis with the comparison between GEM $(5 \mathrm{nM})$ alone and GEM $(5 \mathrm{nM})+$ VPA $(0.5 \mathrm{mM})$ combination.

Table I. Differentially expressed genes by microarray analysis (GEM vs. GEM+VPA).

\begin{tabular}{ll}
\hline & \multicolumn{1}{c}{ Genes } \\
\hline Up-regulated (n=37) & ANKRD1, ANKRD22, CPM, LRRC49, CALB2, SERPINB7, CCDC88A, IQGAP2, CAMK4, ENPP1, \\
& PRKAR2B, SGCE, LRRCC1, HLA-DRA, HLA-DRB1, HLA-DRB3, HLA-DPB1, SELL, PLCE1, \\
& CNTN1, ARHGDIB, SILV, PHOSPHO1, PSG4, ATP6V1C2, MBOAT2, CALCRL, SPP1, SEPP1, \\
& SSBP2, CTGF, TSPAN13, PEG10, CD274, TSPAN7, FHL1, TMPRSS11E \\
Down-regulated (n=6) & HIST2H2BE, DPEP1, LGALS9, ABCA12, LCN2, NMI \\
\hline
\end{tabular}

Assays-on-demand and the TaqMan Universal Master mix (Applied Biosystems). The following assays were used: HDAC1 (no. Hs00606262_m1), HLA-DRA (no. Hs00219575_m1). The TaqMan Human ACTB endogenous control (4326315E) was used as a control gene. The thermal cycler conditions were as follows: $2 \mathrm{~min}$ at $50^{\circ} \mathrm{C}, 10 \mathrm{~min}$ at $95^{\circ} \mathrm{C}, 40$ cycles of $15 \mathrm{sec}$ at $95^{\circ} \mathrm{C}$, and $1 \mathrm{~min}$ at $60^{\circ} \mathrm{C}$. Amplification data were analyzed with an Applied Biosystems Prism 7500 Sequence Detection system version 1.3.1 (Applied Biosystems).

Statistical analyses. Statistical comparisons of mean values were conducted using one-way ANOVA. All the results are presented as mean \pm SD. Statistical analysis was performed using StatView 5.0 J Software (SAS Institute, Inc., Cary, NC, USA). A P-value of $<0.05$ was considered to be statistically significant.

\section{Results}

In the GEM alone group, no effect of GEM was observed in the dose of $5 \mathrm{nM}$. Proliferation-inhibitory effects (16 and $43 \%$ ) were observed at doses of 10 and $50 \mathrm{nM}$ (Fig. 1A). In the VPA alone group, no effect of VPA was observed in the dose of $0.5 \mathrm{mM}$. Proliferation-inhibitory effects $(12,35$ and $67 \%)$ were observed in doses of $1.0,5.0$ and $10 \mathrm{mM}$, respectively (Fig. 1B). GEM (5 nM) combined with VPA (0.5 mM) reduced proliferation by $23 \%$, which significantly augmented the anticancer effects of GEM alone or VPA alone $(\mathrm{P}<0.01)$ (Fig. 1C). In regard to HDAC1 expression, GEM combined with VPA reduced the HDAC1 expression compared to GEM alone or VPA alone with real-time RT-PCR (Fig. 2).

Using microarray analysis, 683 and 357 unique genes were isolated in the GEM $(5 \mathrm{nM})$ and VPA $(0.5 \mathrm{mM})$ groups in comparison to the control group (fold change $>2.0$ ), respectively (data not shown). In addition, 43 differentially expressed genes were isolated between the GEM alone group and the GEM+VPA combination group (fold change >2.0) (Table I).

$\mathrm{GO}$ analysis revealed that the genes of the MHC class II protein complex showed significant different patterns $(\mathrm{P}=0.04)$ between the GEM alone group and the GEM+VPA combination group (Fig. 3). IPA-represented up-regulated (red) and down-regulated (green) genes in the gene network of the 'Cellular Development' contained the gene of the MHC class II protein complex, HLA-DRA, among the isolated genes between the two groups (Fig. 4). 


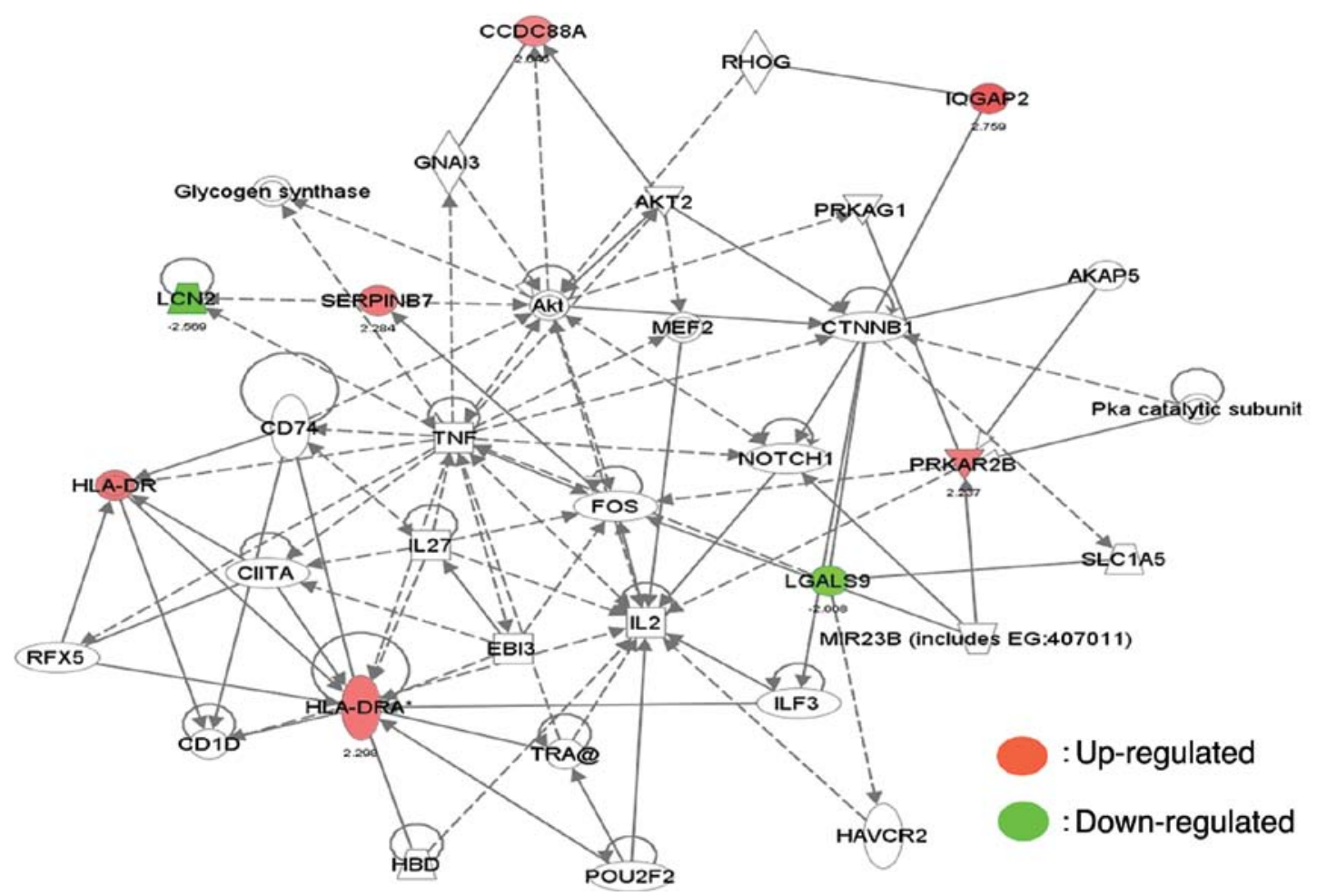

Figure 4. The gene network with the genes isolated from the comparison between GEM (5 nM) alone and GEM (5 nM) + VPA (0.5 mM) using IPA.

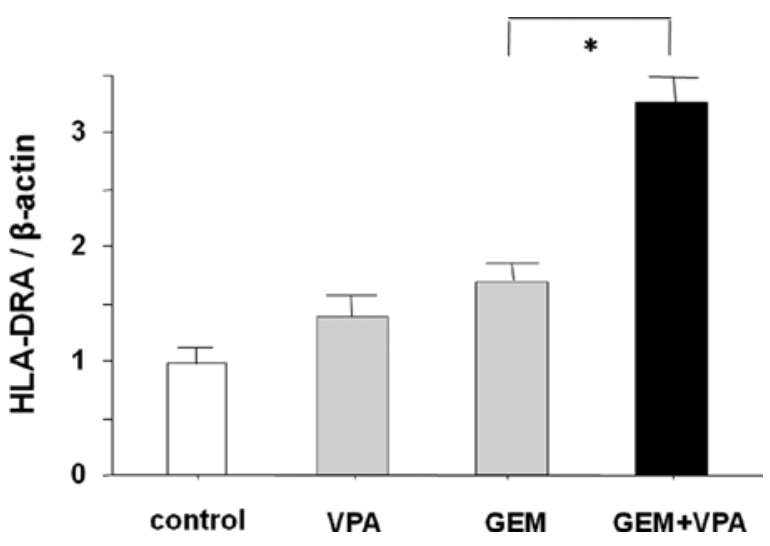

Figure 5. The gene-expressions in the HLA-DRA of human cholangiocarcinoma cell line, HuCCT1, with the combination of GEM $(5 \mathrm{nM})$ and VPA $(0.5 \mathrm{mM}){ }^{*} \mathrm{P}<0.01$.

Furthermore, up-regulation of the HLA-DRA expression in the group of GEM combined with VPA was confirmed by RT-PCR compared to the GEM alone or the VPA alone groups $(\mathrm{P}<0.01)$ (Fig. 5).

\section{Discussion}

In the present study, we assessed the effects of VPA, an HDAC inhibitor, in combination with GEM in the bile duct carcinoma cell line. To the best of our knowledge, this is the first report showing that VPA enhances the effects of GEM in a bile duct carcinoma cell line.
HDAC inhibitors have been shown to be useful in cancer therapy since they sensitize cancer cells to multiple chemotherapeutic agents $(4,14)$. The HDAC inhibitor SAHA has been reported to enhance the effects of GEM in non-small cell lung cancer through the regulation of $\mathrm{NF}-\kappa \mathrm{B}(15)$. In the pancreatic cancer cell line, SAHA has been shown to augment the effects of GEM through up-regulation of p21 expression (16).

In this study, we utilized DNA microarray analysis and IPA to investigate the molecular mechanisms of the anticancer effects of VPA in combination with GEM. The microarray analysis may identify potential target genes for therapy, while IPA can be useful to understand the pathobiology at multiple levels by integrating data from a variety of experimental platforms and providing insight into the molecular and chemical interactions, cellular phenotypes and disease processes. We used IPA to analyze the gene network with the comparison between GEM only and the GEM+VPA combination. Using microarray analysis, VPA enhanced the anticancer effect of GEM through the gene network of the 'Cellular Development' containing HLA-DRA.

MHC class II molecules (HLA-DR, HLA-DP and HLA-DQ in humans) bind and display peptide antigens for recognition by $\mathrm{CD}^{+} \mathrm{T}$ lymphocytes. MHC class II molecules play an important role in antitumor immunity $(17,18)$. The expression of HLA-DR antigen in various cancers has been reported to influence carcinogenesis and prognosis (19-21) and the low expression of HLA-DR is an independent risk factor in HCC (22).

Furthermore, the expression of HLA-DR antigen in gastric cancer is associated with the degree of tumor cell differentiation $(19,20)$. HDAC inhibitors can induce the differentiation of 
solid tumors and reduce cell proliferation (23-25). Inhibition of the cell cycle is an essential event in cellular differentiation and the cytostatic effects of HDAC inhibitors are important for their anticancer activities. Apicidin, an HDAC inhibitor, caused the induction of stem cell differentiation into all three germ layers (26). In view of these findings, HDAC inhibitors have the potential to be used as differentiation-inducing therapy for cancer cells.

Many reports have documented the importance of histone acetylation as a positive regulator of MHC class II transcription $(27,28)$ and HDAC inhibitors augmented the expression of the tumor cell MHC class II (29). On the other hand, HDAC activity repressed the MHC class II gene expression (30). In this study, after comparing the GEM alone group to the GEM and VPA combination group, the interactions were shown between genes of the 'Cellular Development' network containing the gene of the MHC class II protein, HLA-DRA. The HLA-DRA expression was up-regulated in the GEM plus VPA group. VPA may affect the differentiation of bile duct carcinoma cells because of the close correlation already shown between the expression of HLA-DR antigen and the differentiation of tumor cells $(19,20)$. However, some of the HDAC inhibitors are of limited therapeutic use due to their toxic side effects at high doses (31). VPA is widely used as a therapeutic drug for epilepsy and its toxic profile and pharmacokinetic properties are well established. Furthermore, in our study, the promising dose of VPA was $0.5 \mathrm{mM}$, while the peak plasma concentration in patients treated for epilepsy ranging between 0.5 and $1.2 \mathrm{mM}$ (32). VPA, at a dose of $0.5 \mathrm{mM}$, may not cause any serious side effects in clinical settings.

In conclusion, VPA augmented the therapeutic effects of GEM, at least partially by increasing the HLA-DRA expression. These effects may be associated with the modification of cancer cell differentiation.

\section{Acknowledgements}

A part of this study was presented in ASCO-GI, in San Francisco, January 20-22, 2011. Grant support was provided by the Grants-in-Aid for Scientific Research of the Japan Society for the Promotion of Science (Grant-in-Aid for Young Scientists B: No. 22791286). We would like to thank Ms. Harada for providing technical assistance. Regarding supports in microarray analysis, we want to extend our special thanks to Mr. Horikawa, The University of Tokushima Graduate School, Institute of Health Biosciences, and the Support Center for Advanced Medical Sciences.

\section{References}

1. Cereda S, Passoni P, Reni M, et al: The cisplatin, epirubicin, 5-fluorouracil, gemcitabine (PEFG) regimen in advanced biliary tract adenocarcinoma. Cancer 116: 2208-2214, 2010.

2. Shimada M, Sugimoto K, Iwahashi S, et al: CD133 expression is a potential prognostic indicator in intrahepatic cholangiocarcinoma. J Gastroenterol 45: 896-902, 2010.

3. Valle J, Wasan H, Palmer DH, et al: Cisplatin plus gemcitabine versus gemcitabine for biliary tract cancer. N Engl J Med 362: 1273-1281, 2010.

4. Iwahashi S, Ishibashi H, Utsunomiya T, et al: Effect of histone deacetylase inhibitor in combination with 5-fluorouracil on pancreas cancer and cholangiocarcinoma cell lines. J Med Invest 58: 106-109, 2011
5. Haberland M, Montgomery RL and Olson EN: The many roles of histone deacetylases in development and physiology: implications for disease and therapy. Nat Rev Genet 10: 32-42, 2009

6. Patra SK, Patra A and Dahiya R: Histone deacetylase and DNA methyltransferase in human prostate cancer. Biochem Biophys Res Commun 287: 705-713, 2001.

7. Lin RJ, Nagy L, Inoue S, et al: Role of the histone deacetylase complex in acute promyelocytic leukaemia. Nature 391: 811-814, 1998.

8. Miyake K, Yoshizumi T, Imura S, et al: Expression of hypoxia-inducible factor-1alpha, histone deacetylase 1 , and metastasis-associated protein 1 in pancreatic carcinoma: correlation with poor prognosis with possible regulation. Pancreas 36 : e1-e9, 2008.

9. Minucci S and Pelicci PG: Histone deacetylase inhibitors and the promise of epigenetic (and more) treatments for cancer. Nat Rev Cancer 6: 38-51, 2006.

10. Glozak MA and Seto E: Histone deacetylases and cancer. Oncogene 26: 5420-5432, 2007.

11. Göttlicher M, Minucci S, Zhu P, et al: Valproic acid defines a novel class of HDAC inhibitors inducing differentiation of transformed cells. EMBO J 20: 6969-6978, 2001.

12. Jones J, Bentas W, Blaheta RA, et al: Modulation of adhesion and growth of colon and pancreatic cancer cells by the histone deacetylase inhibitor valproic acid. Int J Mol Med 22: 293-299, 2008.

13. Xia Q, Sung J, Chowdhury W, et al: Chronic administration of valproic acid inhibits prostate cancer cell growth in vitro and in vivo. Cancer Res 66: 7237-7244, 2006.

14. Fuino L, Bali P, Wittmann S, et al: Histone deacetylase inhibitor LAQ824 down-regulates Her-2 and sensitizes human breast cancer cells to trastuzumab, taxotere, gemcitabine, and epothilone B. Mol Cancer Ther 2: 971-984, 2003.

15. Rundall BK, Denlinger CE and Jones DR: Suberoylanilide hydroxamic acid combined with gemcitabine enhances apoptosis in non-small cell lung cancer. Surgery 138: 360-367, 2005.

16. Nichole B, Nohea A, Jason G and Murray K: The histone deacetylase inhibitor suberoylanilide hydroxamic acid induces growth inhibition and enhances gemcitabine-induced cell death in pancreatic cancer. Clin Cancer Res 13: 18-26, 2007.

17. Armstrong TD, Clements VK, Martin BK, Ting JP and Ostrand-Rosenberg S: Major histocompatibility complex class II-transfected tumor cells present endogenous antigen and are potent inducers of tumor-specific immunity. Proc Natl Acad Sci USA 94: 6886-6891, 1997.

18. Baskar S, Azarenko V, Garcia Marshall E, Hughes E and OstrandRosenberg S: MHC class II-transfected tumor cells induce long-term tumor-specific immunity in autologous mice. Cell Immunol 155: 123-133, 1994.

19. Ma XC, Hattori T, Kushima R, Terata $N$ and Kodama M: Expression of HLA-class II antigen in gastric carcinomas. Its relationship to histopathological grade, lymphocyte infiltration and five-year survival rate. Acta Oncologica 33: 187-190, 1994.

20. Ishigami S, Aikou T, Natsugoe S, et al: Prognostic value of HLA-DR expression and dendritic cell infiltration in gastric cancer. Oncology 55: 65-69, 1998.

21. Vora AR, Rodgers S, Parker AJ, Start R, Rees RC and Murray AK: An immunohistochemical study of altered immunomodulatory molecule expression in head and neck squamous cell carcinoma. Br J Cancer 76: 836-844, 1997.

22. Matoba K, Iizuka N, Gondo T, et al: Tumor HLA-DR expression linked to early intrahepatic recurrence of hepatocellular carcinoma. Int J Cancer 115: 231-240, 2005.

23. Marks PA, Richon VM and Rifkind RA: Histone deacetylase inhibitors: inducers of differentiation or apoptosis of transformed cells. J Natl Cancer Inst 92: 1210-1216, 2000.

24. Marks P, Rifkind RA, Richon VM, Breslow R, Miller T and Kelly WK: Histone deacetylases and cancer: causes and therapies. Nature Rev Cancer 1: 194-202, 2001.

25. Kramer OH, Gottlicher M and Heinzel T: Histone deacetylase as a therapeutic target. Trends Endocrinol Metab 12: 294-300, 2001.

26. You JS, Kang JK, Seo DW, et al: Depletion of embryonic stem cell signature by histone deacetylase inhibitor in NCCIT cells: involvement of Nanog suppression. Cancer Res 69: 5716-5725, 2009.

27. Beresford GW and Boss JM: CIITA coordinates multiple histone acetylation modifications at the HLA-DRA promoter. Nature Immunol 2: 652-657, 2001. 
28. Masternak K and Reith W: Promoter-specific functions of CIITA and the MHC class II enhanceosome in transcriptional activation. EMBO J 21: 1379-1388, 2002.

29. Niesen MI and Blanck G: Rescue of major histocompatibilityDR surface expression in retinoblastoma-defective, non-small cell lung carcinoma cells by the MS-275 histone deacetylase inhibitor. Biol Pharm Bull 32: 480-482, 2009.

30. Osborne A, Zhang H, Yang WM, Seto E and Blanck G: Histone deacetylase activity represses gamma interferon-inducible HLA-DR gene expression following the establishment of a DNase I-hypersensitive chromatin conformation. Mol Cell Biol 21: 6495-6506, 2001.
31. Warrell RP Jr, He LZ, Richon V, Calleja E and Pandolfi PP: Therapeutic targeting of transcription in acute promyelocytic leukemia by use of an inhibitor of histone deacetylase. J Natl Cancer Inst 90: 1621-1625, 1998.

32. Blaheta RA, Michaelis M, Driever PH and Cinatl J Jr: Evolving anticancer drug valproic acid: insights into the mechanism and clinical studies. Med Res Rev 25: 383-397, 2005. 